\title{
Metric-affine gravity
}

and

\section{the Nester-Witten 2-form}

\author{
Marco Godina*, Paolo Matteucci ${ }^{\dagger \dagger}$ \& James A. Vickers ${ }^{\dagger}$
}

$4^{\text {th }}$ November 2018

\begin{abstract}
In this paper we redefine the well-known metric-affine Hilbert Lagrangian in terms of a spin-connection and a spin-tetrad. On applying the Poincaré-Cartan method and using the geometry of gauge-natural bundles, a global gravitational superpotential is derived. On specializing to the case of the Kosmann lift, we recover the result originally found by Kijowski (1978) for the metric (natural) Hilbert Lagrangian. On choosing a different, suitable lift, we can also recover the NesterWitten 2-form, which plays an important role in the energy positivity proof and in many quasi-local definitions of mass.
\end{abstract}

\section{Introduction}

Conserved quantities have always represented an intriguing issue in general relativity, as was pointed out by Penrose (1982) in a very famous paper. The jet bundle formalism provides an adequate framework for Lagrangian field theories and the Poincaré-Cartan method enables one to associate with each of them globally conserved charges ( $c f .$, e.g., Trautman 1967; Krupka 1971;

*Dipartimento di Matematica, Università di Torino, Via Carlo Alberto 10, 10123 Torino, Italy.

${ }^{\dagger}$ Faculty of Mathematical Studies, University of Southampton, Highfield, Southampton SO17 1BJ, England (UK).

${ }^{\ddagger}$ Corresponding author. E-mail address: p.matteucci@maths.soton.ac.uk. 
Giachetta et al. 1997). In particular, for first order theories these charges are uniquely defined, and in the second order case, although uniqueness is lost, still there is a unique canonical choice.

Natural Lagrangian field theories have been known for a long time, Einstein's general relativity being one of them. Many physical theories, though, such as Yang-Mills and Dirac theories, are non-natural, i.e. the "configuration bundle", which is nothing but the space of the dependent variables or "fields", is not a natural bundle. Roughly speaking, natural bundles (such as the tangent or the cotangent bundle) form a particular class of fibre bundles, where, once a coordinate change on the base manifold is given, the corresponding fibred coordinate change is known. More technically, natural bundles can be regarded as fibre bundles associated with higher order frame bundles on manifolds ( $c f$. Kolář et al. 1993).

If we aim at considering the coupling of a natural theory, such as general relativity, with a non-natural one, we are sometimes forced to "redefine" our field variables in order to make the coupling physically meaningful. In particular, if we want to describe the interaction and feedback between gravity and spinor fields, spin-tetrads, and not tetrads, are the appropriate objects to be considered ( $c f$. Fatibene et al. 1998; Godina et al. 2000). Gauge-natural bundles provide a suitable geometrical framework for such objects. These bundles are fibre bundles associated with "abstract" principal bundles with arbitrary structure group ( $c f$. Kolář et al. 1993).

The superpotential associated with the standard Hilbert Lagrangian for general relativity, or the "Hilbert superpotential", was first given by Kijowski (1978) using ideas developed by Kijowski himself (1973) and Kijowski \& Szczyrba $(1975,1976)$. It was also derived explicitly using the PoincaréCartan method by Kijowski \& Tulczyjew (1979), in a Hamiltonian (multisymplectic) framework, and Ferraris et al. (1986), in the Lagrangian context ${ }^{1}$. In Ferraris et al. (1994) the authors were able to reformulate the previous result of two of them in terms of tetrads. But, again, their theory was still natural, and this meant there was no real advantage of such a reformulation.

Recently, two parallel papers (Fatibene et al. 1998; Godina et al. 2000) addressed the problem of re-expressing the above results in terms of spintetrads and coupling true general relativity with Fermionic matter, but their findings implicitly relied on a Poincaré-Cartan form associated with a particular ("quasi-natural") lift of vector fields onto the bundle of orthonormal frames, the "Kosmann lift" ( $c f$. Fatibene et al. 1996).

\footnotetext{
${ }^{1}$ There exists an extensive literature on both the multisymplectic formulation of field theories and its Lagrangian counterpart. The interested reader is referred to Gotay et al. (1998), on page 15 and $25-6$, respectively
} 
In this paper we redefine the metric-affine Hilbert Lagrangian in terms of a spin-connection and a spin-tetrad. The ensuing superpotential is genuinely "general", in the sense that it is derived in a completely gauge-natural context, and also allows for the presence of torsion.

Such a reformulation enables us not only to single out the aforementioned link between the Hilbert superpotential and the Kosmann lift, but also to associate the well-known Nester-Witten 2-form with another particular lift, thereby providing us with a clear-cut geometric interpretation of a rather famous but somewhat obscure integrand in general relativity. This lift turns out to be essentially the dual of the Kosmann lift. For a different characterization of the Nester-Witten 2-form see, e.g., the detailed analysis by Dubois-Violette \& Madore (1987).

The structure of the paper is as follows: in $\S 1$ we recall the main ingredients of the Poincaré-Cartan method, in $§ 2$ we set up the geometric framework of our theory, and in $\S 3$ we derive our main results.

Finally, in $\S 4$ we present a first order covariant Lagrangian for general relativity and derive the relevant superpotential.

\section{Poincaré-Cartan method}

It is well-know that to each first order Lagrangian there corresponds a unique global Poincaré-Cartan form. Let $M$ be an (orientable, Hausdorff, paracompact, smooth) $m$-dimensional manifold and

$$
\left\{\begin{array}{l}
\mathcal{L}: J^{1} B \rightarrow \bigwedge^{m} T^{*} M \\
\mathcal{L}: j^{1} y \mapsto \mathcal{L}\left(j^{1} y\right) \equiv L\left(x^{\lambda}, y^{\mathfrak{a}}, y^{\mathfrak{a}}{ }_{\mu}\right) \mathrm{d} s
\end{array}\right.
$$

a first order Lagrangian defined on the first order jet prolongation $J^{1} B$ of a gauge-natural bundle $B$ over $M\left(c f\right.$. Kolář et al. 1993, §51), $\left(x^{\lambda}, y^{\mathfrak{a}}\right)$ being coordinates on $B$ and $\mathrm{d} s \equiv \mathrm{d} x^{0} \wedge \mathrm{d} x^{1} \wedge \cdots \wedge \mathrm{d} x^{m-1}$ the natural volume element on $M$. Define its momenta as

$$
f_{\mathfrak{a}}^{\mu}:=\frac{\partial L}{\partial y^{\mathfrak{a}}} .
$$

The Poincaré-Cartan form associated with $\mathcal{L}$ is then given by

$$
\Theta(\mathcal{L}):=\mathcal{L}+f_{\mathfrak{a}}{ }^{\mu} \mathrm{d}_{\mathrm{V}} y^{\mathfrak{a}} \wedge \mathrm{d} s_{\mu}
$$

where $\mathrm{d}_{\mathrm{V}}$ is the vertical differential (notably, $\mathrm{d}_{\mathrm{V}} y^{\mathfrak{a}}=\mathrm{d} y^{\mathfrak{a}}-y_{\mu}^{\mathfrak{a}} \mathrm{d} x^{\mu}$ : cf. Giachetta et al. 1997) and we set $\left.\mathrm{d} s_{\mu}:=\partial_{\mu}\right\rfloor \mathrm{d} s$, '」' denoting the inner product. 
The knowledge of the Poincaré-Cartan form enables us to calculate the socalled Noether current of the Lagrangian in question. Indeed, if one has a one-parameter subgroup of automorphisms of $B$ generated by a projectable vector field $\Xi$ (with projection $\xi$ onto $M$ ), the Noether current associated with $\mathcal{L}$ along the vector field $\Xi$ is given by

$$
\begin{aligned}
E(\mathcal{L}, \Xi): & \left.=-\operatorname{Hor}\left[J^{1} \Xi\right\rfloor \Theta(\mathcal{L})\right] \\
& =-\xi\rfloor \mathcal{L}+f_{\mathfrak{a}}{ }^{\mu} £_{\Xi} y^{\mathfrak{a}} \mathrm{d} s_{\mu}
\end{aligned}
$$

where Hor denotes the horizontal projection ( $c f$. Giachetta et al. 1997, §3.1), $J^{1} \Xi$ is the first order jet prolongation of $\Xi$, and the well-known relation

$$
\left.J^{1} \Xi\right\rfloor \mathrm{d}_{\mathrm{V}} y^{\mathfrak{a}}=-£_{\Xi} y^{\mathfrak{a}}
$$

between vertical differential and (generalized) Lie derivative is used in obtaining the second equation ( $c f$. Kolář et al. 1993, §47).

\section{Geometric framework}

Let $M$ be an orientable, Hausdorff, paracompact, smooth, 4-dimensional manifold. Suppose $M$ admits Lorentzian metrics of signature -2, i.e. assume that $M$ satisfies the topological requirements which ensure the existence on it of Lorentzian structures [SO $(1,3)^{e}$-reductions]. Let $\mathbb{L}(M)$ be the (principal) bundle of linear frames over $M$ with structure group $\mathrm{GL}(4, \mathbb{R})$.

Assume now that $M$ admits a free spin structure $(\Sigma, \tilde{\Lambda})$, i.e. the existence of at least one principal (fibre) bundle $\Sigma$ over $M$ with structure group $\operatorname{Spin}(1,3)^{e} \cong \mathrm{SL}(2, \mathbb{C})$, called the spin structure bundle, and at least one strong (i.e. covering the identity map) equivariant morphism $\tilde{\Lambda}: \Sigma \rightarrow \mathbb{L}(M)$ (Godina et al. 2000). We call the bundle map $\tilde{\Lambda}$ a spin-frame on $\Sigma$.

This definition of a spin structure induces metrics on $M$. Indeed, given a spin-frame $\tilde{\Lambda}: \Sigma \rightarrow \mathbb{L}(M)$, we can define a metric via the reduced subbundle $\mathrm{SO}(M, g) \equiv \tilde{\Lambda}(\Sigma)$ of $\mathbb{L}(M)$. In other words, the dynamic metric $g \equiv g_{\tilde{\Lambda}}$ is defined to be the metric such that frames in $\tilde{\Lambda}(\Sigma) \subset \mathbb{L}(M)$ are $g$-orthonormal frames. It is important to stress that in our picture the metric $g$ is built up a posteriori, after a spin-frame has been determined by the field equations

in a way which is compatible with the (free) spin structure one has used to define spinors.

Now let $\Lambda$ be the epimorphism which exhibits $\operatorname{Spin}(1,3)^{e}$ as a twofold covering of $\mathrm{SO}(1,3)^{e}$ and consider the following left action of the group 
$\mathrm{GL}(4, \mathbb{R}) \times \operatorname{Spin}(1,3)^{e}$ on the manifold $\mathrm{GL}(4, \mathbb{R})$

$$
\left\{\begin{array}{l}
\rho:\left(\mathrm{GL}(4, \mathbb{R}) \times \operatorname{Spin}(1,3)^{e}\right) \times \mathrm{GL}(4, \mathbb{R}) \rightarrow \mathrm{GL}(4, \mathbb{R}) \\
\rho:\left(\left(A^{\mu}{ }_{\nu}, S^{a}{ }_{b}\right), u_{\mu}^{a}\right) \mapsto u^{\prime a}{ }_{\mu}:=(\Lambda(S))^{a}{ }_{b} u_{\nu}^{b}\left(A^{-1}\right)^{\nu}{ }_{\mu}
\end{array}\right.
$$

together with the associated bundle $\Sigma_{\rho}:=W^{1,0}(\Sigma) \times{ }_{\rho} \operatorname{GL}(4, \mathbb{R})$, where $W^{1,0}(\Sigma):=\mathbb{L}(M) \underset{M}{\times} \Sigma$ denotes the principal prolongation of order $(1,0)$ of the

principal fibre bundle $\Sigma$ (cf. Kolář et al. 1993, §52.4). The bundle $W^{1,0}(\Sigma)$ is a principal fibre bundle with structure group $\mathrm{GL}(4, \mathbb{R}) \times \operatorname{Spin}(1,3)^{e}$. It turns out that $\Sigma_{\rho}$ is a fibre bundle associated with $W^{1,0}(\Sigma)$, i.e. a gauge-natural bundle of order $(1,0)$. A section of $\Sigma_{\rho}$ will be called a spin-tetrad.

Recall now that a (principal) connection on a principal (fibre) bundle $P(M, G)$ may be regarded as a $G$-equivariant global section of the affine jet bundle $J^{1} P \rightarrow P$, where the $G$-action on $J^{1} P$ is induced by the first jet prolongation of the canonical (right) action of $G$ on $P$ ( $c f$. Giachetta et al. $1997, \S 2.7)$. Owing to $G$-equivariance there is a $1-1$ correspondence between principal connections and global sections of the quotient bundle $J^{1} P / G \rightarrow M$.

More specifically, let $P=\Sigma$ and let $\mathfrak{s p i n}(1,3) \cong \mathfrak{s o}(1,3) \cong \mathfrak{s l}(2, \mathbb{C})$ denote the Lie algebra of $\operatorname{Spin}(1,3)^{e}$. Consider then the following left action on the vector space $V_{C}:=\left(\mathbb{R}^{4}\right)^{*} \otimes \mathfrak{s o}(1,3)$

$$
\left\{\begin{array}{l}
\lambda:\left(\mathrm{GL}(4, \mathbb{R}) \times T_{4}^{1} \operatorname{Spin}(1,3)^{e}\right) \times V_{C} \rightarrow V_{C} \\
\lambda:\left(\left(A_{\nu}^{\mu}, S_{b}^{a}, S_{b \mu}^{a}\right), u_{b \mu}^{a}\right) \mapsto u_{b \mu}^{\prime a}:=\left(A^{-1}\right)^{\nu}{ }_{\mu}\left[(\Lambda(S))^{a}{ }_{c} u_{d \nu}^{c}\left(\Lambda\left(S^{-1}\right)\right)^{d}{ }_{b}\right. \\
\left.-(\Lambda(S))^{a}{ }_{c \nu}\left(\Lambda\left(S^{-1}\right)\right)_{b}^{c}\right]
\end{array},\right.
$$

where $(\Lambda(S))_{c \nu}^{a}$ are the components of $j_{0}^{1}(\Lambda \circ S)$, an element of $T_{4}^{1} \mathrm{SO}(1,3)^{e}$, and $S: \mathbb{R}^{4} \rightarrow \operatorname{Spin}(1,3)^{e}$ is a local map defined around the origin $0 \in \mathbb{R}^{4}$. Hence define the associated bundle $C:=W^{1,1}(\Sigma) \times_{\lambda} V_{C}$, where $W^{1,1}(\Sigma):=$ $\mathbb{L}(M) \underset{M}{\times} J^{1} \Sigma$ denotes the principal prolongation of order $(1,1)$ of $\Sigma(c f$. Kolár et al. $1993, \S 52.4)$. It turns out that $C$ is a gauge-natural bundle of order $(1,1)$ isomorphic to $J^{1}\left(\Sigma / \mathbb{Z}_{2}\right) / \mathrm{SO}(1,3)^{e}$. A section of $C$ will be called a spinconnection.

\section{$3 \quad$ Metric-affine gravity}

Let $\theta^{a}{ }_{\mu}$ be a spin-tetrad and $\omega_{b \mu}^{a}$ a spin-connection, as defined in the previous section. Set locally

$$
\begin{aligned}
\theta^{a} & :=\theta^{a}{ }_{\mu} \mathrm{d} x^{\mu}, \\
e_{a} & :=e_{a}{ }^{\mu} \partial_{\mu},
\end{aligned}
$$


where $e_{a}{ }^{\mu}$ is implicitly defined via the relation $\theta^{a}{ }_{\mu} e_{b}{ }^{\mu}=\delta_{b}^{a}$, and

$$
\begin{aligned}
\omega_{b}^{a} & :=\omega_{b \mu}^{a} \mathrm{~d} x^{\mu}, \\
\Omega_{b}^{a}{ }_{b} & :=\mathrm{d}_{\mathrm{H}} \omega^{a}{ }_{b}+\omega^{a}{ }_{c} \wedge \omega_{b}^{c},
\end{aligned}
$$

$\mathrm{d}_{\mathrm{H}}$ being the horizontal differential ( $c f$. Giachetta et al. 1997, §3.1); $\omega^{a}{ }_{b}$ and $\Omega^{a}{ }_{b}$ are recognized to be the (horizontal) connection 1-form and curvature 2-form, respectively.

We can now "redefine" the (metric-affine) Hilbert Lagrangian as

$$
\left\{\begin{array}{l}
\mathcal{L}: \Sigma_{\rho} \underset{M}{\times} J^{1} C \rightarrow \wedge^{4} T^{*} M \\
\mathcal{L}:\left(\theta^{a}{ }_{\mu}, j^{1} \omega^{a}{ }_{b \mu}\right) \mapsto \mathcal{L}\left(\theta^{a}{ }_{\mu}, j^{1} \omega^{a}{ }_{b \mu}\right):=-\frac{1}{2 \kappa} \Omega_{a b} \wedge \Sigma^{a b}
\end{array},\right.
$$

where $\kappa:=8 \pi G / c^{4}$ and $\Sigma^{a b}:={ }^{*}\left(\theta^{a} \wedge \theta^{b}\right)$. The equations of motion are obtained by varying $\mathcal{L}$ with respect to $\theta^{c}$ and $\omega_{a b}$ :

$$
\begin{aligned}
\frac{\delta \mathcal{L}}{\delta \theta^{c}} & \equiv \frac{1}{2 \kappa} \Omega_{a b} \wedge \Sigma^{a b}{ }_{c} \equiv-\frac{1}{\kappa} G^{a}{ }_{c} \Sigma_{a}=0 \\
\frac{\delta \mathcal{L}}{\delta \omega_{a b}} & \equiv \frac{1}{2 \kappa} \nabla \Sigma^{a b}=0
\end{aligned}
$$

where $\left.\Sigma_{c}^{a b}{ }_{c}:=e_{c}\right\rfloor \Sigma^{a b}, \Sigma_{a}:=1 / 6 e_{a b c d} \theta^{b} \wedge \theta^{c} \wedge \theta^{d}$ and $\nabla$ denotes the (gauge-) covariant exterior derivative. We stress that the condition $\nabla \Sigma^{a b}=0$ is equivalent to $T^{a} \equiv \nabla \theta^{a}=0, T^{a}$ being the torsion 2 -form.

According to the definition given in $\S 1$, the appropriate Poincaré-Cartan form for Lagrangian (3.1) is

$$
\begin{aligned}
\Theta(\mathcal{L}) & \equiv \mathcal{L}+\mathrm{d}_{\mathrm{V}} \omega_{a b} \wedge \frac{\partial \mathcal{L}}{\partial \mathrm{d}_{\mathrm{H}} \omega_{a b}} \\
& =\mathcal{L}-\frac{1}{2 \kappa} \mathrm{d}_{\mathrm{V}} \omega_{a b} \wedge \Sigma^{a b}
\end{aligned}
$$

where $\partial \mathcal{L} / \partial \mathrm{d}_{\mathrm{H}} \omega_{a b}$ stands for $\partial L / \partial \omega_{a b \nu, \mu} \mathrm{d} s_{\mu \nu}$ and $\left.\mathrm{d} s_{\mu \nu}:=\partial_{\nu}\right\rfloor \mathrm{d} s_{\mu}$. Hence, the Noether current associated with a projectable vector field $\Xi$ is

$$
\begin{aligned}
E(\mathcal{L}, \Xi) & =-\xi\rfloor \mathcal{L}-\frac{1}{2 \kappa} £_{\Xi} \omega_{a b} \wedge \Sigma^{a b} \\
& \left.\left.\equiv \frac{1}{2 \kappa}\left[(\xi\rfloor \Omega_{a b}\right) \wedge \Sigma^{a b}+\Omega_{a b} \wedge(\xi\rfloor \Sigma^{a b}\right)-£_{\Xi} \omega_{a b} \wedge \Sigma^{a b}\right] \\
& \left.\equiv \frac{1}{2 \kappa}\left[(\xi\rfloor \Omega_{a b}\right) \wedge \Sigma^{a b}+\xi^{c} \Omega_{a b} \wedge \Sigma^{a b}{ }_{c}-£_{\Xi} \omega_{a b} \wedge \Sigma^{a b}\right] .
\end{aligned}
$$

Now, our configuration bundle $B$ is $\Sigma_{\rho} \times C$, which is a gauge-natural bundle. Therefore, every (principal) automorphism $\Phi \in \operatorname{Aut}(\Sigma)$ induces an automorphism $\Phi_{B}$ on $B$. This holds also infinitesimally, i.e. for invariant (projectable) vector fields defined on $\Sigma$. Strictly speaking, an invariant vector field $\Xi \in \mathfrak{X}(\Sigma)$ defines functorially a projectable vector field $\Xi_{B} \in \mathfrak{X}\left(\Sigma_{\rho} \times{ }_{M} C\right)$. 
Moreover, every $\operatorname{Spin}(1,3)^{e}$-invariant vector field $\Xi \in \mathfrak{X}(\Sigma)$ projects onto an $\mathrm{SO}(1,3)^{e}$-invariant vector field, which we denote by the same symbol $\Xi \in \mathfrak{X}\left(\Sigma / \mathbb{Z}_{2}\right)$. Since the natural projection pr: $\Sigma \rightarrow \Sigma / \mathbb{Z}_{2}$ is a covering map (locally, a diffeomorphism) of principal fibre bundles, it follows that there is a bijection between projectable $\mathrm{SO}(1,3)^{e}$-invariant vector fields on $\Sigma / \mathbb{Z}_{2}$ and projectable $\operatorname{Spin}(1,3)^{e}$-invariant vector fields on $\Sigma$ ( $c f$. Fatibene et al. 1996). If a spin-frame is given, such a bijection extends to an invariant vector field bijection between $\Sigma / \mathbb{Z}_{2}$ and $\mathrm{SO}(M, g) \equiv \tilde{\Lambda}(\Sigma)$, and, hence, between $\mathrm{SO}(M, g)$ and $\Sigma$. Yet, only the Lie derivative of the connection 1-form is needed here, so we can simply regard $\Xi_{B}$ as belonging to $\mathfrak{X}(C)$. Then, a projectable vector field $\Xi_{C} \in \mathfrak{X}(C)$ onto a vector field $\xi \equiv \xi^{\mu} \partial_{\mu} \in \mathfrak{X}(M)$ reads as

$$
\Xi_{C}=\xi^{\mu} \partial_{\mu}+\Xi_{b \mu}^{a} \frac{\partial}{\partial u_{b \mu}^{a}}
$$

where

$$
\Xi_{b \mu}^{a}:=-\left(\partial_{\mu} \xi^{\nu} u_{b \nu}^{a}+u_{c \mu}^{a} \Xi_{b}^{c}-u_{b \mu}^{c} \Xi_{c}^{a}+\partial_{\mu} \Xi_{b}^{a}\right),
$$

$\Xi \equiv \xi^{\mu}(x) \partial_{\mu}+\Xi_{b}^{a}(x) \alpha_{a}{ }^{b}$ being the corresponding projectable vector field on $\Sigma / \mathbb{Z}_{2}$ and $\left(u_{b \mu}^{a}\right)$ local fibre coordinates on $C$. The vector fields $\alpha_{a}{ }^{b}$ are local right $\mathrm{SO}(1,3)^{e}$-invariant vector fields on $\Sigma / \mathbb{Z}_{2}$, which in a suitable chart $\left(x^{\mu}, u_{a}^{b}\right)$ read as

$$
\alpha_{a}{ }^{b} \equiv \frac{1}{2}\left(\rho_{a}{ }^{b}-\eta^{b c} \eta_{a d} \rho_{c}{ }^{d}\right),
$$

$\eta$ denoting the Minkowski metric and $\rho_{a}{ }^{b}:=u_{c}{ }^{b} \partial / \partial u_{c}{ }^{a}$. Therefore, the Lie derivative of $u_{b \mu}^{a}=\omega^{a}{ }_{b \mu}(x)$ is just

$$
£_{\Xi} \omega_{b \mu}^{a}=\xi^{\nu} \partial_{\nu} \omega_{b \mu}^{a}+\partial_{\mu} \xi^{\nu} \omega_{b \nu}^{a}+\omega_{c \mu}^{a} \Xi_{b}^{c}-\omega_{b \mu}^{c} \Xi_{c}^{a}+\partial_{\mu} \Xi_{b}^{a},
$$

which can be readily recast in Cartan formalism as

$$
\left.£_{\Xi} \omega_{b}^{a}=\xi\right\rfloor \Omega_{b}^{a}+\nabla \check{\Xi}_{b}^{a},
$$

$\check{\Xi}_{b}^{a}{ }_{b}:=\Xi^{a}{ }_{b}+\omega_{b \mu}^{a} \xi^{\mu}$ being the vertical part of $\Xi$. On substituting (3.5) into (3.4), we finally get

$$
\begin{aligned}
E(\mathcal{L}, \Xi) & =\frac{1}{2 \kappa}\left(\xi^{c} \Omega_{a b} \wedge \Sigma^{a b}{ }_{c}-\nabla \check{\Xi}_{a b} \wedge \Sigma^{a b}\right) \\
& =\frac{1}{2 \kappa}\left[\xi^{c} \Omega_{a b} \wedge \Sigma^{a b}{ }_{c}+\check{\Xi}_{a b} \nabla \Sigma^{a b}-\mathrm{d}_{\mathrm{H}}\left(\check{\Xi}_{a b} \Sigma^{a b}\right)\right] .
\end{aligned}
$$


Now, by virtue of equations of motion $(3.2 a)$ and $(3.2 b)$,

$$
U(\mathcal{L}, \Xi):=-\frac{1}{2 \kappa} \check{\Xi}_{a b} \Sigma^{a b}
$$

is recognized to be the superpotential associated with Lagrangian (3.1). This superpotential, which was derived in a completely gauge-natural context and - to the best of our knowledge - appears here for the first time, represents the most general superpotential possible in this metric-affine formulation of gravity (modulo, of course, closed 2-forms).

Note that in the case of the Kosmann lift (Fatibene et al. 1996) we have

$$
\check{\Xi}_{a b}=\left(\check{\xi}_{\mathrm{K}}\right)_{a b} \equiv-\nabla_{[a} \xi_{b]},
$$

which, substituted in (3.7), gives

$$
U\left(\mathcal{L}, \xi_{\mathrm{K}}\right)=\frac{1}{2 \kappa} \nabla_{a} \xi_{b} \Sigma^{a b}
$$

i.e. half of the well-known Komar (1959) potential, in accordance with the result found by Ferraris et al. (1994) in a purely natural context. This is also the lift implicitly used by Godina et al. (2000) in the 2-spinor formalism.

Let now $\sigma_{a}{ }^{A A^{\prime}}$ denote the Infeld-van der Waerden symbols, which express the isomorphism between $\operatorname{Re}[S(M) \otimes \bar{S}(M)]$ and $T M$ in the orthonormal basis induced by the spin-frame chosen ( $c f$. Penrose \& Rindler 1984; Wald 1984), and consider the following lift:

$$
\xi^{\mu}=e_{a}{ }^{\mu} \sigma_{A A^{\prime}}^{a} \lambda^{A} \bar{\lambda}^{A^{\prime}}, \quad \check{\Xi}_{a b}=\left(\check{\xi}_{\mathrm{W}}\right)_{a b}:=-4 \sigma_{[a}^{A A^{\prime}} \sigma_{b]}{ }^{B B^{\prime}} \operatorname{Re}\left(\bar{\lambda}_{B^{\prime}} \nabla_{B A^{\prime}} \lambda_{A}\right),
$$

which will be referred to as the Witten lift. Then

$$
U\left(\mathcal{L}, \xi_{\mathrm{W}}\right)=\operatorname{Re} W \equiv-\frac{2}{\kappa} \operatorname{Re}\left(\mathrm{i} \bar{\lambda}_{A^{\prime}} \nabla \lambda_{A} \wedge \theta^{A A^{\prime}}\right),
$$

which is the (real) Nester-Witten 2-form (Nester 1981; Penrose \& Rindler 1986). Indeed, we have ${ }^{2}$ :

$$
\begin{aligned}
\check{\Xi}_{a b} \Sigma^{a b} & =-2 \bar{\lambda}_{B^{\prime}} \nabla_{B A^{\prime}} \lambda_{A} \Sigma^{a b}+\mathrm{CC} \\
& =2 \mathrm{i}^{*}\left(\bar{\lambda}_{A^{\prime}} \nabla_{B B^{\prime}} \lambda_{A}\right) \Sigma^{a b}+\mathrm{CC} \\
& =2 \mathrm{i} \bar{\lambda}_{A^{\prime}} \nabla_{b} \lambda_{A}{ }^{*} \Sigma^{a b}+\mathrm{CC} \\
& =-2 \mathrm{i} \bar{\lambda}_{A^{\prime}} \nabla_{b} \lambda_{A} \theta^{a} \wedge \theta^{b}+\mathrm{CC} \\
& =2 \mathrm{i} \bar{\lambda}_{A^{\prime}} \nabla \lambda_{A} \wedge \theta^{A A^{\prime}}+\mathrm{CC},
\end{aligned}
$$

\footnotetext{
${ }^{2}$ With the exception of formula (3.13) below, we shall suppress hereafter the Infeld-van der Waerden symbols and adopt the standard identification $a=A A^{\prime}, b=B B^{\prime}$, \&c., as is customary in the current literature ( $c f$. Penrose \& Rindler 1984).
} 
where we used the identities ( $c f$. Penrose \& Rindler 1984)

$$
{ }^{*} A_{a b} B^{a b}=A_{a b}{ }^{*} B^{a b}, \quad{ }^{* *} A^{a b}=-A^{a b}, \quad{ }^{*} A^{A B A^{\prime} B^{\prime}}=\mathrm{i} A^{A B B^{\prime} A^{\prime}}
$$

for any two bivectors $A^{a b}$ and $B^{a b}$. Inserting (3.12) into (3.7) gives (3.11), as claimed.

If we wish, it is also possible to define a complexified Witten lift as

$$
\xi^{\mu}=e_{a}{ }^{\mu} \sigma^{a}{ }_{A A^{\prime}} \lambda^{A} \bar{\lambda}^{A^{\prime}}, \quad \check{\Xi}_{a b}=\left(\check{\xi}_{\mathrm{W}}^{\mathbb{C}}\right)_{a b}:=-4 \sigma_{[a}{ }^{A A^{\prime}} \sigma_{b]}{ }^{B B^{\prime}} \bar{\lambda}_{B^{\prime}} \nabla_{B A^{\prime}} \lambda_{A}
$$

Then, the relevant superpotential is

$$
U\left(\mathcal{L}, \xi_{\mathrm{W}}^{\mathbb{C}}\right)=W:=-\frac{2 \mathrm{i}}{\kappa} \bar{\lambda}_{A^{\prime}} \nabla \lambda_{A} \wedge \theta^{A A^{\prime}}
$$

which is the (complex) Nester-Witten 2-form (Penrose \& Rindler 1986; Mason \& Frauendiener 1990). From the viewpoint of physical applications (proof of positivity of the Bondi or ADM mass, quasi-local definitions of momentum and angular momentum in general relativity, \&c.), it is immaterial whether one uses (3.14) or its real part (3.11), as its imaginary part turns out to be $-1 / \kappa \mathrm{d}_{\mathrm{H}}\left(\lambda_{A} \bar{\lambda}_{A^{\prime}} \theta^{a}\right)$, which vanishes upon integration over a closed 2-surface. Note, though, that (3.14) appears to relate more directly to Penrose quasilocal 4-momentum, when suitable identifications are made ( $c f$. Penrose \& Rindler 1986, p. 432).

Remark 3.1. Note also that - modulo an inessential numerical factor-the Kosmann lift is (the real part of) the dual of the (complex) Witten lift, in the sense that

$$
\left(\check{\xi}_{\mathrm{K}}\right)_{a b}=-\frac{1}{2} \operatorname{Re}\left[{ }^{*}\left(\check{\xi}_{\mathrm{W}}^{\mathbb{C}}\right)_{a b}\right]
$$

as can be easily checked on starting from equations (3.8) and (3.13)(2), whenever, of course, $\xi^{a}=\lambda^{A} \bar{\lambda}^{A^{\prime}}$.

Remark 3.2. The theory developed herein is obviously tailored to the coupling with spinor fields described by the Dirac Lagrangian,

$$
\mathcal{L}_{\mathrm{D}}:=\left[\frac{\mathrm{i}}{2}\left(\tilde{\Psi} \gamma^{a} \nabla_{a} \Psi-\widetilde{\nabla_{a} \Psi} \gamma^{a} \Psi\right)-m \tilde{\Psi} \Psi\right] \sqrt{g} \mathrm{~d} s .
$$

In the purely metric case, the total superpotential turns out to be

$$
U\left(\mathcal{L}+\mathcal{L}_{\mathrm{D}}, \Xi\right)=U(\mathcal{L}, \Xi)+U\left(\mathcal{L}_{\mathrm{D}}, \Xi\right)
$$


where

$$
\begin{aligned}
U\left(\mathcal{L}_{\mathrm{D}}, \Xi\right): & =\frac{\mathrm{i}}{8} \tilde{\Psi}\left[\left(\gamma_{a} \gamma_{b} \gamma_{c}+2 \eta_{a c} \gamma_{b}\right) \xi^{c}\right] \Psi \Sigma^{a b}, \\
& \equiv \frac{\mathrm{i} \sqrt{2}}{4} \xi_{A}^{A^{\prime}}\left(\bar{\varphi}_{A^{\prime}} \varphi_{B}-\bar{\psi}_{B} \psi_{A^{\prime}}\right) \Sigma^{A B}+\mathrm{CC}
\end{aligned}
$$

The reader is referred to Fatibene et al. (1998) and Godina et al. (2000) for further details and notation.

Conversely, in the present metric-affine context, it can be readily shown that, although the Dirac Lagrangian does enter the equations of motion (notably, the "second" Einstein-Cartan equation), it does not contribute to the total superpotential. From this fact one might mistakenly conclude that the Dirac fields do not contribute to the total conserved quantities. This conclusion is wrong because, although the Dirac Lagrangian does not contribute directly to the superpotential, in order to obtain the corresponding conserved quantities, one needs integrate the superpotential on a solution, which in turn depends on the Dirac Lagrangian via its energy-momentum tensor and the second Einstein-Cartan equation.

\section{First order gravity}

In the case of vanishing torsion $\left(T^{a} \equiv 0 \Longleftrightarrow \nabla \Sigma^{a b} \equiv 0\right)$, it is easy to see that Lagrangian (3.1) can be split into a total divergence plus a first order covariant Lagrangian. In many contexts, the superpotential associated with this Lagrangian proved to give more physically reasonable answers than the Hilbert superpotential ( $c f$. Godina et al. 2000).

For this reason and the sake of completeness, we now give the derivation of the aforementioned superpotential in the new geometrical framework outlined in $\S 2$.

The first order covariant Lagrangian in question is (cf. Ferraris \& Francaviglia 1990; Ferraris et al. 1994)

$$
\begin{aligned}
\hat{\mathcal{L}} & :=-\frac{1}{2 \kappa}\left(\hat{\Omega}_{a b}-Q_{a c} \wedge Q_{b}^{c}\right) \wedge \Sigma^{a b} \\
& \equiv \mathcal{L}+\frac{1}{2 \kappa} \mathrm{d}_{\mathrm{H}}\left(Q_{a b} \wedge \Sigma^{a b}\right),
\end{aligned}
$$

where $\mathcal{L}$ is given by (3.1), $\hat{\Omega}_{a b}:=\mathrm{d}_{\mathrm{H}} \hat{\omega}_{a b}+\hat{\omega}_{a c} \wedge \hat{\omega}_{b}^{c}$ and $Q_{a b}:=\omega_{a b}-\hat{\omega}_{a b}, \hat{\omega}_{a b}$ being a "background" (non-dynamical) spin-connection. The corresponding Poincaré-Cartan form is

$$
\begin{aligned}
\Theta(\hat{\mathcal{L}}) & =\hat{\mathcal{L}}-\frac{1}{2 \kappa}\left(\mathrm{d}_{\mathrm{V}} \hat{\omega}_{a b} \wedge \Sigma^{a b}-\mathrm{d}_{\mathrm{V}} \Sigma^{a b} \wedge Q_{a b}\right) \\
& \equiv \Theta(\mathcal{L})+\frac{1}{2 \kappa}\left[\mathrm{d}_{\mathrm{H}}\left(Q_{a b} \wedge \Sigma^{a b}\right)+\mathrm{d}_{\mathrm{V}} Q_{a b} \wedge \Sigma^{a b}+\mathrm{d}_{\mathrm{V}} \Sigma^{a b} \wedge Q_{a b}\right]
\end{aligned}
$$


Hence, the Noether current associated with a projectable vector field $\Xi$ is

$$
\begin{aligned}
\left.E(\hat{\mathcal{L}}, \Xi)=E(\mathcal{L}, \Xi)+\frac{1}{2 \kappa}\left[£_{\Xi} Q_{a b} \wedge \Sigma^{a b}+£_{\Xi} \Sigma^{a b} \wedge Q_{a b}-\xi\right\rfloor \mathrm{d}_{\mathrm{H}}\left(Q_{a b} \wedge \Sigma^{a b}\right)\right] \\
=E(\mathcal{L}, \Xi)+\frac{1}{2 \kappa}\left[£_{\Xi} Q_{a b} \wedge \Sigma^{a b}+£_{\Xi}\left(Q_{a b} \wedge \Sigma^{a b}\right)-£_{\Xi} Q_{a b} \wedge \Sigma^{a b}\right. \\
\left.\quad-\xi\rfloor \mathrm{d}_{\mathrm{H}}\left(Q_{a b} \wedge \Sigma^{a b}\right)\right] \\
\left.=E(\mathcal{L}, \Xi)+\frac{1}{2 \kappa}\left[£_{\Xi}\left(Q_{a b} \wedge \Sigma^{a b}\right)-\xi\right\rfloor \mathrm{d}_{\mathrm{H}}\left(Q_{a b} \wedge \Sigma^{a b}\right)\right]
\end{aligned}
$$

$\xi$ denoting, as usual, the projection of $\Xi$ onto $M$. Now,

$$
\begin{aligned}
£_{\Xi}\left(Q_{a b} \wedge \Sigma^{a b}\right) & \equiv £_{\xi}\left(Q_{a b} \wedge \Sigma^{a b}\right) \\
& \left.=\xi\rfloor \mathrm{d}_{\mathrm{H}}\left(Q_{a b} \wedge \Sigma^{a b}\right)+\mathrm{d}_{\mathrm{H}}[\xi\rfloor\left(Q_{a b} \wedge \Sigma^{a b}\right)\right] .
\end{aligned}
$$

On substituting (4.3) into (4.2), we get

$$
\left.E(\hat{\mathcal{L}}, \Xi)=E(\mathcal{L}, \Xi)+\frac{1}{2 \kappa} \mathrm{d}_{\mathrm{H}}[\xi\rfloor\left(Q_{a b} \wedge \Sigma^{a b}\right)\right]
$$

whence

$$
\left.U(\hat{\mathcal{L}}, \Xi):=U(\mathcal{L}, \Xi)+\frac{1}{2 \kappa} \xi\right\rfloor\left(Q_{a b} \wedge \Sigma^{a b}\right)
$$

is recognized to be the superpotential associated with Lagrangian (4.1).

Remark 4.1. Note that, contrary to what happens in the purely natural context, no additional conditions need be imposed on the vector field $\Xi$ here.

\section{Discussion}

This paper stresses the important role the theory of gauge-natural bundles plays in a significant issue of mathematical physics such as the definition of the gravitational energy and, more generally, of conserved quantities associated with the gravitational field, especially when coupled to spinor fields.

Besides providing a new gravitational superpotential in a gauge-natural context, the paper sheds some new light on the definition of the NesterWitten 2-form and gives it an interpretation as a further, genuine gravitational superpotential.

Moreover, this paper shows that it is crucial in this context not to regard the metric as the fundamental gravitational field. Indeed, when considering the interaction between gravity and spinors one is forced to give up a purely natural formalism and instead consider a gauge-natural formalism in which one chooses a spin-tetrad (together with a spin-connection, in a metric-affine formulation) as one's fundamental variable. 
A parallel and analogous method of investigation is possible when dealing, in a gauge-natural context, with Legendre and dual Legendre transforms, for which the reader is referred to the recent and fundamental papers by Raiteri et al. (1996) and Ferraris et al. (2000).

\section{Acknowledgements}

P. M. acknowledges an EPSRC research studentship and a Faculty Research Studentship from the University of Southampton.

\section{References}

Dubois-Violette, M. \& Madore, J. (1987). 'Conservation laws and integrability conditions for gravitational and Yang-Mills field equations'. Comm. Math. Phys. 108, 213-23.

Fatibene, L., Ferraris, M., Francaviglia, M. \& Godina, M. (1996). 'A geometric definition of Lie derivative for spinor fields'. In J. Janyška, I. Kolár \& J. Slovák (Eds.), Proc. 6th International Conference on Differential Geometry and its Applications (Brno, 1995), pp. 549-58. Brno: Masaryk University.

Fatibene, L., Ferraris, M., Francaviglia, M. \& Godina, M. (1998). 'Gauge formalism for general relativity and fermionic matter'. Gen. Rel. Grav. 30(9), 1371-89.

Ferraris, M. \& Francaviglia, M. (1990). 'Covariant first-order Lagrangians, energy-density and superpotentials in general relativity'. Gen. Rel. Grav. $22(9), 965-85$.

Ferraris, M., Francaviglia, M. \& Mottini, M. (1994). 'Conserved quantities of the gravitational field in tetrad notation'. Rend. Mat. Appl. (7) 14, $457-81$.

Ferraris, M., Francaviglia, M. \& Raiteri, M. (2000). 'Dual Lagrangian field theories'. J. Math. Phys. 41(4), 1889-915.

Ferraris, M., Francaviglia, M. \& Robutti, O. (1986). 'Energy and superpotentials in gravitational field theories'. In M. Modugno (Ed.), Atti del $6^{o}$ Convegno Nazionale di Relatività Generale e Fisica della Gravitazione, pp. 137-50. Bologna: Pitagora Editrice. 
Giachetta, G., Mangiarotti, L. \& Sardanashvily, G. (1997). New Lagrangian and Hamiltonian methods in field theory. Singapore: World Scientific.

Godina, M., Matteucci, P., Fatibene, L. \& Francaviglia, M. (2000). 'Twospinor formulation of first order gravity coupled to Dirac fields'. Gen. Rel. Grav. 32(1), 145-59.

Gotay, M. J., Isenberg, J. \& Marsden, J. E. (1998). 'Momentum maps and classical relativistic fields. Part I: Covariant field theory'. E-print: physics@xxx.lanl.gov (physics/9801019).

Kijowski, J. (1973). 'A finite-dimensional canonical formalism in the classical field theory'. Comm. Math. Phys. 30, 99-128.

Kijowski, J. (1978). 'On a new variational principle in general relativity and the energy of the gravitational field'. Gen. Rel. Grav. 9(10), 857-77.

Kijowski, J. \& Szczyrba, W. (1975). 'Multisymplectic manifolds and the geometrical construction of the Poisson brackets in the classical field theory'. In J.-M. Souriau (Ed.), Géométrie Symplectique et Physique Mathématique (Colloq. Internat. C.N.R.S., Aix-en-Provence, 1974), pp. 347-79. Paris: C.N.R.S.

Kijowski, J. \& Szczyrba, W. (1976). 'A canonical structure for classical field theories'. Comm. Math. Phys. 46, 183-206.

Kijowski, J. \& Tulczyjew, W. M. (1979). A symplectic framework for field theories. Lecture Notes in Physics 107. Berlin: Springer-Verlag.

Kolár, I., Michor, P. W. \& Slovák, J. (1993). Natural operations in differential geometry. Berlin: Springer-Verlag.

Komar, A. (1959). 'Covariant conservation laws in general relativity'. Phys. Rev. 113, 934-6.

Krupka, D. (1971). 'Lagrange theory in fibered manifolds'. Rep. Mathematical Phys. 2, 121-33.

Mason, L. J. \& Frauendiener, J. (1990). 'The Sparling 3-form, Ashtekar variables and quasi-local mass'. In T. N. Bailey \& R. J. Baston (Eds.), Twistors in mathematics and physics, London Math. Soc. Lecture Notes 156, pp. 189-217. Cambridge: Cambridge University Press.

Nester, J. M. (1981). 'A new gravitational energy expression with a simple positivity proof'. Phys. Lett. 83A, 241-2. 
Penrose, R. (1982). 'Quasi-local mass and angular momentum in general relativity'. Proc. Roy. Soc. London Ser. A 381, 53-62.

Penrose, R. \& Rindler, W. (1984). Spinors and space-time. Vol. 1: Twospinor calculus and relativistic fields. Cambridge: Cambridge University Press.

Penrose, R. \& Rindler, W. (1986). Spinors and space-time. Vol. 2: Spinor and twistor methods in space-time geometry. Cambridge: Cambridge University Press.

Raiteri, M., Ferraris, M. \& Francaviglia, M. (1996). 'General relativity as a gauge theory of orthogonal groups in three dimensions'. In P. Pronin \& G. Sardanashvily (Eds.), Gravity, particles and space-time, pp. 81-98. Singapore: World Scientific.

Trautman, A. (1967). 'Noether equations and conservation laws'. Comm. Math. Phys. 6, 248-61.

Wald, R. M. (1984). General relativity. Chicago: The University of Chicago Press. 